\title{
AN APPROACH FOR MODELING OF A MEDICAL EQUIPMENT FOR THE ESTIMATION OF LEAKAGE CURRENTS
}

\author{
E. ZENNARO ${ }^{1}$, G.L. AMICUCCI ${ }^{2}$, F. FIAMINGO ${ }^{2}$ \& C. MAZZETTI ${ }^{1}$ \\ ${ }^{1}$ Department of Astronautics, Electrical and Energetics Engineering (DIAEE), Electrical Engineering Section, \\ Università di Roma 'La Sapienza', Italy. \\ ${ }^{2}$ Department of Safety Technology (DTS), Research, Certification and Verification Sector, \\ INAIL - Istituto Nazionale per l'Assicurazione contro gli Infortuni sul Lavoro, Italy.
}

\begin{abstract}
In an operating theatre, electro medical equipment (EME) may fail and cause health hazards as the passing of a weak but hazardous current (leakage current) through the heart of the patient during surgical interventions. This occurs evidently during their use, when connected to the patient. The related values of leakage currents can be estimated by an electrical circuit model. To obtain the circuit of a surgical layout, the electrical models of medical location supply power system, human body and EME involved in a determined surgical intervention ought to be drawn. The present work focuses on the method to obtain the model of EME. The idea is to model by circuits each leakage current measurement set-up performed in accordance with the international standard IEC 60601-1. To assign the values to the model parameters, it is necessary to obtain also some information on the EME as the values of insulation impedances and the feasible leakage current paths inside it. The case of a commercial defibrillator is taken as an example to show the feasibility of the method. The comparison between the leakage currents simulated by the circuit and the ones measured is here presented. The agreement is satisfactory. An estimation of the model sensitivity due to the uncertainty in the knowledge of the parameters has been performed too, by using the Monte Carlo method. The extension of this approach to draw the model of other EME is also considered in view of the realization of a surgical layout circuit.

Keywords: Applied part, defibrillator circuit model, electrical safety, medical equipment, microshock risk, leakage currents.
\end{abstract}

\section{INTRODUCTION}

As established in literature [1-3], the circulation of a low current (decades of milliampere) through the human body is able to cause respiratory paralysis or potentially fatal ventricular fibrillation. In some medical procedures, the risk of electric shock under faulty conditions increases due to the low resistance pathway via skin penetration and to the decreased patient defences caused by medication or anesthesia $[4,5]$. During open heart surgery and when catheters are in use, it is necessary to minimize leakage of current that may flow into the patient. The critical values in such cases are significantly lower (decades of microampere) than those able to harm in the normal situations. The safety standard IEC EN 60601-1 [6] recommends $50 \mu \mathrm{A}$ as the maximum value admitted for the current flowing from patient via the patient connections of a cardiac floating-type applied part to earth (patient leakage current) in a faulty EME. Limit values in Ref. [6] are established on a series of studies (see, for instance, Watson et al. [7]). However, since such threshold limits are fixed on the base of a probabilistic investigation for a large fraction of population, some authors consider as possibly dangerous also lesser values $[8,9]$. In fact, currents $<50 \mu \mathrm{A}$ could be dangerous for patients with some heart diseases during cardiac surgical procedures.

Indeed, a major problem exists in reconstructing equipment and connection conditions after an incident has occurred, but the most often cited reasons for patient injury in the 
operating theatres are lack of standardization in wiring practices, as well as user's errors and improper use of medical devices [10].

Improper use and user's errors can be reduced through training, information and procedures, while a better wiring practice is obtained following suitable requirements for the electrical system [11].

In fact, isolated power supply systems (medical IT systems or IPS) and equipotential supplementary bonding are standard solutions adopted in group 2 locations as safeguards against microshock [11-13].

In healthcare facilities, to guarantee the safety of the patient and of the medical staff, it is required a periodic assessment of the electrical risk.

The quantitative risk assessment is based on the estimation of the damage probability and magnitude [12]. The estimation of damage probability is possible if the value of the leakage current occurring during a surgical procedure is known. The standard IEC 60601-1 recommends to perform some electrical safety tests to periodically verify the status of EME. These tests consist in measuring the leakage currents in normal and simulated faulty conditions and the insulation resistances. Such measurements are carried out by supplying the EME with AC voltage in a laboratory. Even if periodic tests, improvements in design, more reliable components and the adoption and carrying out of the risk management process would decrease the occurrence of faults, the EME continue to fail leading to health hazards. This occurs evidently during their use, when connected to the patient. Values of leakage current caused by a determined fault involving EME and/or the IPS can be evaluated by a circuit model of the surgical layout. It is composed by electrical circuits of IPS, the patient's human body and EME involved in the considered surgical procedure.

The present contribution proposes an approach to obtain the electrical model of EME. It consists in modeling by circuits each leakage current measurement set-up performed in accordance with [6]. The layout of the circuit should be composed by

- Impedances representing the insulations.

- The load resistance of the EME, reading the load current absorption measured during test.

- The ideal voltage source representing the supply voltage of EME during test.

- The resistance of the measuring device (MD) through which flow the leakage current under exam.

- The high input impedance of the MD.

The insulations of EME are constituted by parallels of resistances and capacitances because it is put into operation at $\mathrm{AC}$ voltage. Hence, a problem arises, since also insulation capacitances should be considered when one settles a model for determining believable values of the leakage currents. To obtain by simulations the same leakage currents measured during test, it is important to identify both the paths of such leakage currents and values of electrical model parameters.

Actually, insulation capacitances are often not available neither in the datasheets of the EME. For such reason to draw a circuit model for simulation purposes, three steps have been followed:

- to measure resistances by using DC voltage;

- to measure leakage currents by using AC voltage and

- to obtain capacitances by reversing mathematical formulas and by using the measured resistances and leakage currents, as shown in Section 4. 
Since the standard EN ISO 14971 [14] suggests to do a quantitative risk assessment by using data from a variety of sources, then test measurements on a real EME, a defibrillator, have been used to identify the parameters of its circuit model and to verify the feasibility of the method. In literature, there exist already examples of electrical models of generic EME for the simulation of possible electrical faults (see, for instance, Spalding et al. [15]), but a method to obtain the model of specific commercial EME operating in surgical theatre is presented here.

\section{THE METHOD FOR THE EME CIRCUIT MODEL DERIVATION}

The standard IEC EN 60601-1 [6] is the key reference for modeling of leakage current measurement set-up of EME.

To obtain the layout of this circuit, some considerations have been made on the electrical layout of EME. It has been determined considering the electrical insulations, which the standard IEC EN 62353 [16] recommend to measure. As described there, the measurement points between which must be placed the DC voltage generator are:

a. the mains part and protective earth for grounded equipment (class I);

b. the mains part and (non-earthed) accessible conductive parts for class I and ungrounded equipment (class II);

c. the mains part and the applied parts;

d. floating type applied part and protective earth for class I equipment;

b. floating type applied part and (non-earthed) accessible conductive parts for class I and class II equipment.

During leakage current tests, EME is supplied at mains frequency $(50 \mathrm{~Hz}$ is the frequency in Europe) and $230 \mathrm{~V} \mathrm{AC}$. When the frequency of the power supply is not null, then the insulation capacitance affects the current circulation.

The measured value of the load current is taken into consideration by the evaluation of the load resistance. Moreover, the capacitances installed by the manufacturer as filter to mitigate the electromagnetic interferences are also represented to evaluate their contribution in the protection of patient from the hazards of intolerable values of leakage current.

The electrical layout of a class I EME with non-earthed accessible conductive parts and with floating type applied part is proposed in Fig. 1.

The circuit shown in Fig. 1 refers to a phase-neutral layout, with floating type isolated (F-type) applied part (AP). The following notes explain the different parts:

- $\boldsymbol{M} \boldsymbol{P}_{1}$ and $\boldsymbol{M P _ { 2 }}$ : mains parts or live (phase and neutral) conductors.

- $\boldsymbol{E}$ : enclosure, i.e. the conductive accessible part that is not protectively earthed and is isolated from the mains parts.

- $\boldsymbol{G}$ : ground, i.e. the conductive accessible part that is protectively earthed by protective earth (PE) conductor and isolated from the mains parts by a basic insulation.

- $\boldsymbol{A P}$ : applied part, i.e. the part that comes into physical contact with the patient for the EME to perform its function.

- $C_{1}, C_{2}$ : capacitances forming the filter for the protection from electromagnetic disturbances (EMI filter).

- $\boldsymbol{R}_{\boldsymbol{I}}$ : EME resistive load, which absorbs electrical power.

- $\boldsymbol{R}_{i 1}, \boldsymbol{C}_{i 1}$ and $\boldsymbol{R}_{i 5}, \boldsymbol{C}_{i 5}$ : basic insulation between live conductors and ground.

- $\boldsymbol{R}_{i 2}, \boldsymbol{C}_{i 2}$ and $\boldsymbol{R}_{i 4}, \boldsymbol{C}_{i 4}$ : insulation between live conductors and enclosure. It should be double 

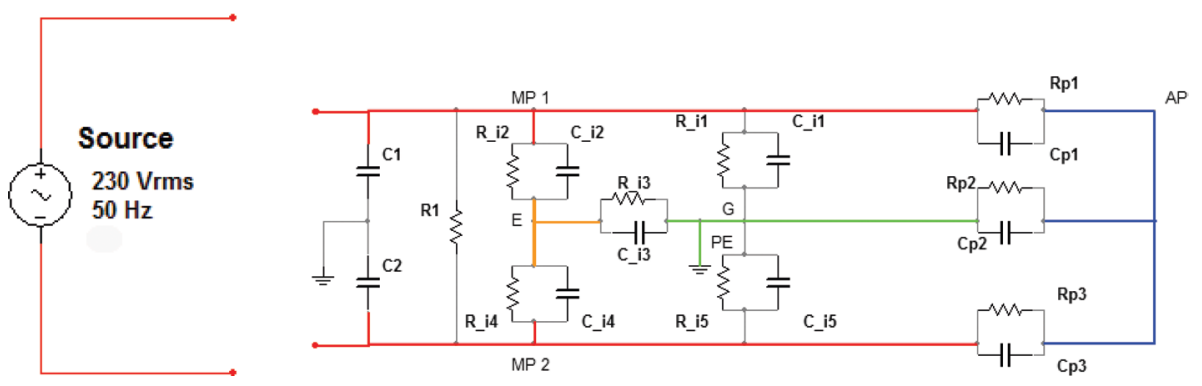

Figure 1: Electrical layout of a class I EME with non-earthed accessible conductive parts and with floating type applied part (AP).

or reinforced in the cases in which the PE connection is not recommended (for example: moving parts in which the reliability of the connection can be poor or class II equipment).

- $\boldsymbol{R}_{i 3}, \boldsymbol{C}_{i 3}$ : insulation between ground and enclosure, this insulation is in addition to the insulations interposed to live conductors.

- $\boldsymbol{R}_{p 1}, \boldsymbol{C}_{p 1}$ and $\boldsymbol{R}_{p 2}, \boldsymbol{C}_{p 2}$ and $\boldsymbol{R}_{p 3}, \boldsymbol{C}_{p 3}$ : insulations between, respectively, applied part and the phase conductor, applied part and ground, applied part and the neutral conductor.

To identify the model parameter values in the case of a real equipment, the circuit model described in Fig. 1 has been applied to obtain the circuit model of a commercial defibrillator. In particular, the leakage current measurement set-up carried on the defibrillator is modeled to simulate leakage currents similar to that measured on the real equipment. Moreover, the leakage current paths are studied, as shown in the next section.

\section{THE METHOD FOR THE DEFIBRILLATOR CIRCUIT MODEL DERIVATION}

The electrical diagram of a commercial defibrillator is obtained starting from the circuit shown in Fig. 1. The number of applied parts and the value of the load current have been obtained by the datasheet, whereas the leakage current measurement set-up compliant with [6] is adopted to obtain reasonable values to assign to the resistances and capacitances composing the model. Fig. 2 shows the measurement set-up of the patient leakage current from an applied part in normal condition, whereas Fig. 3 shows the circuit model of the commercial defibrillator obtained on the base of set-up represented in Fig. 2 and modeling the patient leakage current test from a cardiac floating type applied part (CF). The gray-scaled bands help to understand the parts of the measurement set-up, respectively, represented by the two schemes.

In detail, the defibrillator under test is a class I EME, with a basic insulation between live conductors and ground. $V_{1}$ is the root mean square value of the supply voltage of the defibrillator. The values of leakage currents flowing from enclosure, excluding patient connections, through an external path other than the protective earth conductor, to earth or another part of enclosure (touch current) measured during tests have shown that all conductive accessible parts are protectively earthed. The defibrillator is composed by three $\mathrm{CF}$ - type applied parts and two body floating (BF)-type applied parts. The CF-type applied parts are the ElectroCardioGraphic (ECG) electrodes (called shortly 'electrodes' in the following) and the BF-type applied parts are the defibrillator electrodes (called shortly 'paddles') from which the electrical discharge is delivered for the resuscitation of the patient. Since the circuit with 


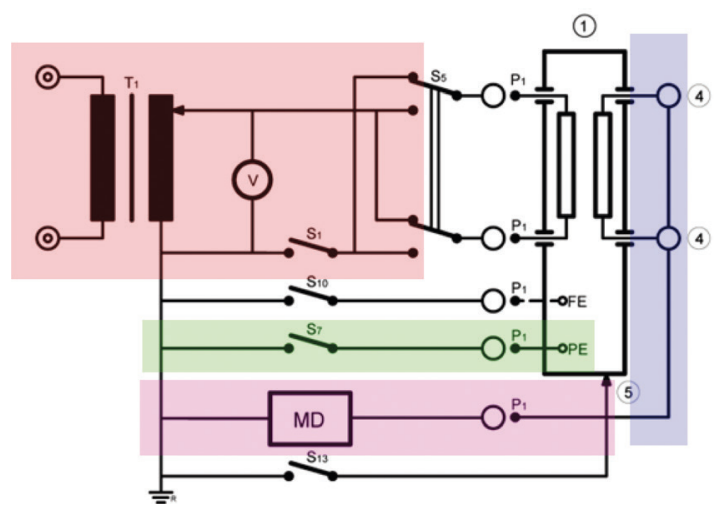

1) EME enclosure;

4) Patient connections;

5) Metal accessibile part not protectively earthed;

P1: Sockets, plugs or terminals for the supply connection of the EME;

T1: Single or polyphase isolation transformer;

S1, S7: Single-pole switches simulating the interruption of a power supply conductor and the protective earth conductor respectively;

S10, S13: Switches for connecting a functional earth terminal to the earthed point of the measuring supply system and for connecting to earth a metal accessible part not protectively earthed;

S5: Commutator switch to reverse the polarity of the mains voltage.

Figure 2: Measurement set-up of the patient leakage current test schematized in the IEC 60601-1. The legend is an extraction of the legend reported in [6].

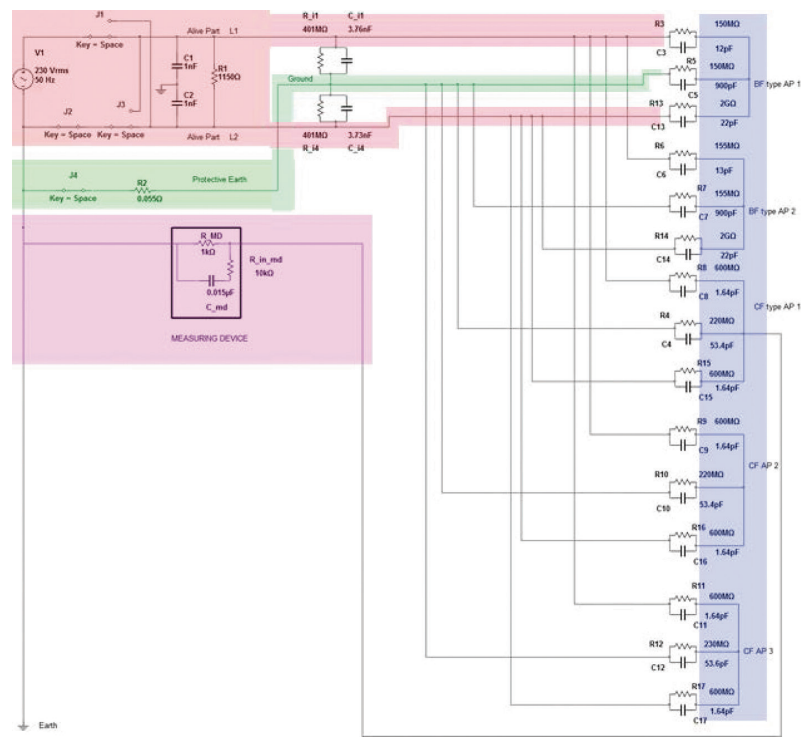

Figure 3: Circuit model of the commercial defibrillator complying with the IEC 60601-1.

the five applied parts was not easily readable, a simplified model could be adopted, as it is shown in Fig. 4. This was done by grouping the applied parts, which perform the same function. In the case of the circuit model of the defibrillator, five applied parts have been considered but only two functions are different, namely the ECG monitoring and electrical shock discharge. Therefore, the scheme can be drawn, without affecting the leakage current simulation, with only two applied parts: one of them represents the ECG electrodes and the other one the paddles.

In Fig. 4, it is shown that the MD is modeled with $1 \mathrm{k} \Omega$ resistance to simulate the conventional value of the human body resistance, and with an high input impedance composed by a series of a $10 \mathrm{k} \Omega$ resistance with a $15 \mathrm{nF}$ capacitance. These values of input impedance are 


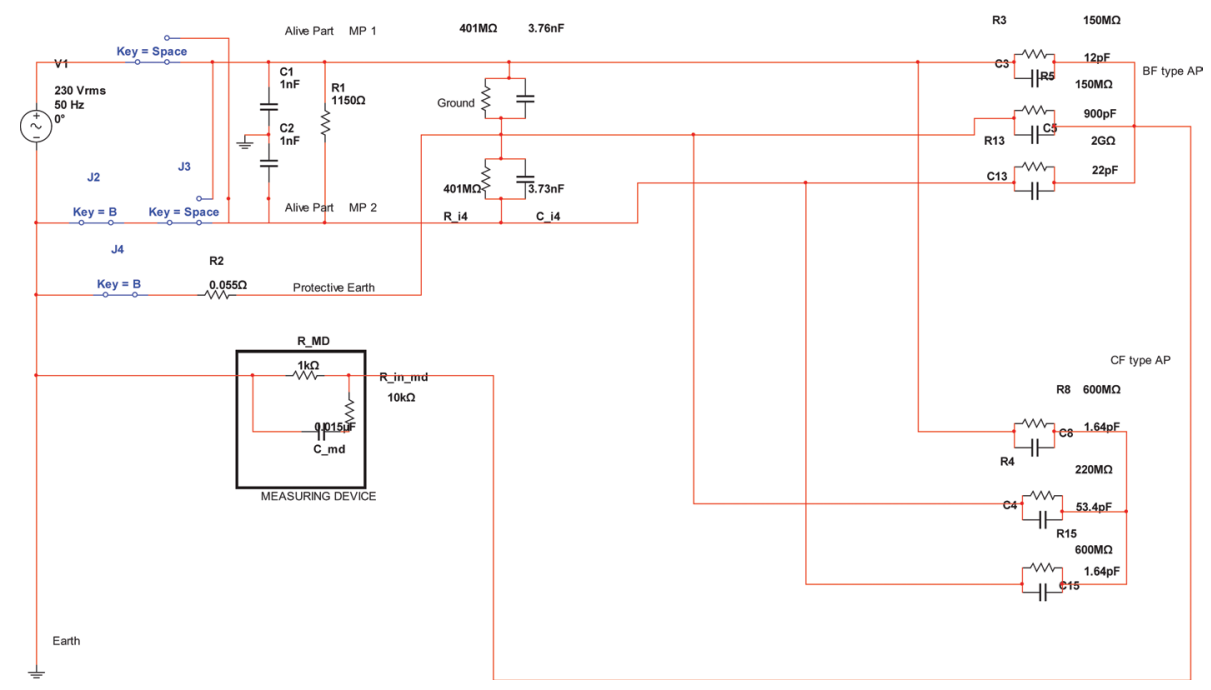

Figure 4: Simplified model of the defibrillator circuit reported in Fig. 3.

common to the most measurement instruments, as digital oscilloscopes, and are compliant with the values required by the standard [6].

The defibrillator has been simulated on a laboratory bench where currents are flowing from the mains part through or across the insulation into the protective earth conductor (earth leakage current), through the enclosure, and across the applied part insulations. These currents are measured in:

- Normal conditions (NC), where all protective measures are in operation; switches in the model are closed.

- Single fault conditions (SFC), when either a supply conductor or the protective earth conductor are open. It means that either switch J2 or switch J4 is open.

- Both with normal supply polarity and reversed supply polarity. It means that switches J1 and $\mathrm{J} 3$ commutate the inversion of polarity.

Each condition is represented by its own circuit. The study of any circuit permits to identify the current path, and the measured value of current is taken into consideration for the evaluation of the electrical parameters, as it is discussed in the next section.

\section{MODEL PARAMETERS IDENTIFICATION}

The identification of the model parameters is done as follows.

Insulation impedance is evaluated by the ratio of root mean square of the voltage drop across the insulation and the root mean square of the current flowing through the insulation. This current is measured as requested by IEC EN 60601-1 [6]. The knowledge of the current path, of the drop voltage and the measurement of related current give the value of impedance. For example, to calculate the basic insulation impedance presented in Fig. 4, and composed by the parallel between $R_{i l}$ and $C_{i l}$, the earth leakage current has been measured. In fact, the current path starts from one phase conductor originated from the voltage source, it continues across the insulation impedance and protective earth conductor resistance and it closes to the 
generator. The knowledge of $V_{\text {rms }}(230 \mathrm{~V})$ and the measured value of $I_{\text {rms }}$ (the earth leakage current in the example) permit to estimate the insulation impedance modulus $|Z|$, as it is reported in eqn (1)

$$
|Z|=\frac{V_{\mathrm{rms}}}{I_{\mathrm{rms}}}
$$

Another mathematical expression for the evaluation of impedance is reported in eqn (2)

$$
Z=\left(\frac{1}{R}+\imath \omega C\right)^{-1}=\frac{R}{1+\imath \omega C R} \Rightarrow \frac{R}{1+\imath \omega C R} \cdot \frac{1-\imath \omega C R}{1-\imath \omega C R}=\frac{R}{1+(\omega C R)^{2}}-\imath \frac{\omega C R^{2}}{1+(\omega C R)^{2}}
$$

It follows eqn (3) to calculate the modulus of insulation impedance

$$
|Z|=\sqrt{\left(\frac{R}{1+(\omega C R)^{2}}\right)^{2}+\left(-\frac{\omega C R^{2}}{1+(\omega C R)^{2}}\right)^{2}}
$$

The value of insulation capacitances have been obtained by using the measured insulation resistances and the calculated insulation impedances by reversing eqn (3) as it is reported in the following equation:

$$
C=\sqrt{\frac{-\left(2|Z|^{2} \omega^{2} R^{2}-\omega^{2} R^{4}\right) \pm \sqrt{\left(2|Z|^{2} \omega^{2} R^{2}-\omega^{2} R^{4}\right)^{2}-4\left(\omega^{4} R^{4}|Z|^{2}\right)\left(|Z|^{2}-R^{2}\right)}}{2 \omega^{4} R^{4}|Z|^{2}}}
$$

For example, the insulation capacitance between ground and the BF-type applied part, $C_{5}$, can be estimated by means of eqn (4), by measuring the insulation resistance $R_{5}$ and by the estimation of the insulation impedance composed by the parallel between $R_{5}$ and $C_{5}$, using eqn (1).

The parameters of the circuit model are shown in Table 1 where the comparison between the electrical parameters of the real defibrillator and those assigned to the model is reported. The abbreviations RA and LL mean 'right arm' and 'left leg' because the MD inlets are suitable for the connection with the electrocardiography electrodes for the measurement of related leakage currents.

As to be noted, the measurement report we have received does not contain the measurement of each resistance value. In those cases, a tuning of the values of insulation resistances has been made considering the aim of the method, namely, to obtain a current flowing through the MD resistance $R_{M D}$ very close to the measured value. It means that the simulated current has to be contained into the accuracy range of the commercial MD used. The measurements were performed by a MD whose accuracy is equal to $1 \%$ of read value $\pm 1 \mu \mathrm{A}$. The tuning of insulation resistances value between the applied part and mains part $\left(R_{3}, R_{13}, R_{6}, R_{14}, R_{8}, R_{15}\right.$, $R_{9}, R_{16}, R_{11}, R_{17}$ in Fig. 3 ) has given outputs compliant with the constraints represented by the measured values of the current flowing through $R_{M D}$ in 20 different tests where the patient leakage current is measured from each of five applied parts in four different conditions, namely normal condition, disconnection of a phase conductor and both states during normal and reversed supply polarity.

As noted in Fig. 4, the EMI filter capacitances are assigned equal to $1 \mathrm{nF}$. This value is reasonable in electronic devices and it only influences the value of current absorbed by the load. 
Table 1: Comparison between the electrical parameters of a real defibrillator and those assigned to the model.

\begin{tabular}{|c|c|c|}
\hline Electrical parameter & Real defibrillator & Simulated defibrillator \\
\hline Load resistance $R_{1}$ & $1150 \Omega$ & $1150 \Omega$ \\
\hline $\mathrm{PE}$ & $0.055 \Omega$ & $0.055 \Omega$ \\
\hline Basic insulation resistance $R_{i 1,4}$ & $\begin{array}{l}\text { (> } 400 \mathrm{M} \Omega \text { maximum of } \\
\text { the measurement range) }\end{array}$ & $401 \mathrm{M} \Omega$ \\
\hline Basic insulation capacitance $C_{i 1,4}$ & Unknown & $\begin{array}{l}3.76 \mathrm{nF} \text { with respect to } \mathrm{MP}_{1} \\
3.73 \mathrm{nF} \text { with respect to } \mathrm{MP}_{2}\end{array}$ \\
\hline $\begin{array}{l}\text { LL paddle insulation resistance } \\
\text { with respect to ground } R_{5}\end{array}$ & $150 \mathrm{M} \Omega$ & $150 \mathrm{M} \Omega$ \\
\hline $\begin{array}{l}\text { LL paddle insulation capacitance } \\
\text { with respect to ground } C_{5}\end{array}$ & Unknown & $900 \mathrm{pF}$ \\
\hline $\begin{array}{l}\text { LL paddle insulation resistance } \\
\text { with respect to live parts } R_{3,13}\end{array}$ & Unknown & $\begin{array}{l}150 \mathrm{M} \Omega \text { with respect to } \\
\mathrm{MP}_{1} \\
2 \mathrm{G} \Omega \text { with respect to } \mathrm{MP}_{2}\end{array}$ \\
\hline $\begin{array}{l}\text { LL paddle insulation capacitance } \\
\text { with respect to live parts } C_{3,13}\end{array}$ & Unknown & $\begin{array}{l}12 \mathrm{pF} \text { with respect to } \mathrm{MP}_{1} \\
22 \mathrm{pF} \text { with respect to } \mathrm{MP}_{2}\end{array}$ \\
\hline $\begin{array}{l}\text { RA electrode insulation resistance } \\
\text { with respect to ground } R_{4}\end{array}$ & $220 \mathrm{M} \Omega$ & $220 \mathrm{M} \Omega$ \\
\hline $\begin{array}{l}\text { RA electrode insulation capaci- } \\
\text { tance with respect to ground } C_{4}\end{array}$ & Unknown & $53.4 \mathrm{pF}$ \\
\hline $\begin{array}{l}\text { RA electrode insulation resistance } \\
\text { with respect to live parts } R_{8,15}\end{array}$ & Unknown & $\begin{array}{l}600 \mathrm{M} \Omega \text { with respect to live } \\
\text { parts }\end{array}$ \\
\hline $\begin{array}{l}\text { RA electrode insulation capaci- } \\
\text { tance with respect to live parts } \\
C_{8,15}\end{array}$ & Unknown & $1.64 \mathrm{pF}$ \\
\hline
\end{tabular}

The values of the electrical parameters of the circuit model in Fig. 4 and reported in Table 1 are identified on the base of the simulations of leakage currents measured during a single campaign of measurements carried on the defibrillator. The uncertainty of the output of the model due to the uncertainty in the estimation of input model parameters has been performed. The procedure adopted for the analysis has been the adaptive Monte Carlo method (MCM) [17] and the results are reported in the next section.

\section{RESULTS AND DISCUSSION}

The simulated leakage current values are reported in Table 2 where they are also compared with the measurement data obtained from tests carried out using a commercial defibrillator. The table shows an example of the measured and simulated values for leakage current in several measurement conditions recommended by the IEC EN 60601-1 [6]. The difference between the experimental and simulated results is satisfactory, contained in the range accuracy of the MD.

The data obtained by the model are in line with what was expected. In fact, the earth leakage current, the touch current and the patient leakage currents in SFC exhibit values higher than those found in normal condition. 
Table 2: Comparison between measured leakage current values of a commercial defibrillator and values obtained from its simulated circuit model.

Device's component

Operating condition

PE

Earth leakage current in normal condition

PE

Earth leakage current when a supply conductor is open

PE

Earth leakage current in normal condition and supply polarity reversed

PE

Earth leakage current when a supply conductor is open and supply polarity reversed

Enclosure

Touch current in normal condition

Enclosure

Touch current when a supply conductor is open

Enclosure

Touch current when PE is open

Enclosure

Touch current in normal condition and supply polarity reversed

Enclosure

Touch current when a supply conductor is open and supply polarity reversed

Enclosure

Touch current when PE is open and supply polarity reversed

Electrode RA

Patient leakage current in normal condition

Electrode RA

Patient leakage current when a supply conductor is open

Electrode RA

Patient leakage current when PE is open

Electrode RA

Patient leakage current in normal condition and supply polarity reversed

Electrode RA

Patient leakage current when a supply conductor is open and supply polarity reversed

Electrode RA

Patient leakage current when PE is open and supply polarity reversed

Paddle LL

Patient leakage current in normal condition
Measured Simulated

value $(\mu \mathrm{A})$ value $(\mu \mathrm{A})$

274

274

522

273

0

0.015

0

0.03

270

0

0.015

0

0.03

273

0

0.4

0

0.8

2

0

0.4

0

0.8

2

2

1.76 
Table 2: Continued

\begin{tabular}{lcc}
\hline $\begin{array}{l}\text { Device's component } \\
\text { Operating condition }\end{array}$ & $\begin{array}{c}\text { Measured } \\
\text { value }(\mu \mathrm{A})\end{array}$ & $\begin{array}{c}\text { Simulated } \\
\text { value }(\mu \mathrm{A})\end{array}$ \\
\hline $\begin{array}{l}\text { Paddle LL } \\
\text { Patient leakage current when a supply conductor is open }\end{array}$ & 2.96 \\
$\begin{array}{l}\text { Paddle LL } \\
\text { Patient leakage current when PE is open }\end{array}$ & 30 & 30.1 \\
$\begin{array}{l}\text { Paddle LL } \\
\text { Patient leakage current in normal condition and supply polarity } \\
\text { reversed }\end{array}$ & 2 & 1.59 \\
$\begin{array}{l}\text { Paddle LL } \\
\text { Patient leakage current when a supply conductor is open and } \\
\quad \text { supply polarity reversed }\end{array}$ & 2 & 2.96 \\
$\begin{array}{l}\text { Paddle LL } \\
\text { Patient leakage current when PE is open and supply polarity } \\
\text { reversed }\end{array}$ & 31 & 30.6 \\
\hline
\end{tabular}

As can be noticed, the simulated patient leakage currents from the paddles, when a supply conductor is open, are not twice the same currents as in normal condition, as it happens for the simulated patient leakage currents from the electrodes.

Moreover, the values of these currents are slightly different when the polarity is reversed. The asymmetry values of the insulation impedances of the paddles with respect to the two live conductors, as shown in Table 1, justify this difference. The importance of the correct estimation of the model input parameters justifies a sensitivity analysis. The results of this analysis performed by MCM take evidence that a variation of $\pm 10 \%$ of parameter values influences the leakage current values approximately of $\pm 10 \%$. It can be concluded that the obtained electrical circuit model of a commercial defibrillator can be considered robust.

\section{CONCLUSION}

A method is presented to obtain the circuit model of an EME. This method consists in the modeling of leakage currents measurement set-up compliant with the requirements of the IEC 60601-1. The study of the electrical layout of the EME under consideration is the first step of the method, in particular the knowledge of how the values of insulation resistances are distributed. To know the model parameters values, the method was applied to the case of real commercial defibrillator to obtain the related electrical circuit.

The estimation of the model sensitivity due to the uncertainty in the knowledge of the parameters, performed by the MCM, has allowed to verify that for a variation of $\pm 10 \%$ of parameter values, the leakage current values range approximately between the same percentage.

The model has been applied to simulate leakage currents from defibrillator in normal condition and in single fault conditions.

The simulated leakage currents are in quite satisfactory agreement with the real ones, taking into account the accuracy range of the MD used for tests.

The procedure used to construct the circuit model of defibrillator can be extended to any other EME after that the electrical circuit layout has been carefully investigated by studying 
the technical datasheet on the light of IEC 60601-1. Then, from this analysis it is possible to know the class of the EME (I or II) and the number and type of applied parts (floating or grounded). The assignment of the values of insulation impedances is obtained from the results of laboratory tests.

The model of an EME is important to build an electrical circuit model of a complete and complex surgical procedure with the aim to identify the most hazardous events leading to the circulation of currents across the patient.

\section{ACKNOWLEDGEMENT}

The authors are grateful to the Clinical Engineering Unit of University Campus Bio-Medico in Rome for his valid support during the campaign of measurements.

\section{REFERENCES}

[1] Ferris, L.P., King, B.G., Spence, P.W. \& Williams, H.B., Effect of electric shock on the heart. Transactions of the American Institute of Electrical Engineers, 55(5), pp. 498-515, 1936. doi: http://dx.doi.org/10.1109/T-AIEE.1936.5057302

[2] Dalziel, C.F. \& Lee, W.R., Reevaluation of lethal electric currents. IEEE Transactions on Industry and General Applications, 5, pp. 467-476, 1968. doi: http://dx.doi. org/10.1109/TIGA.1968.4180929

[3] IEC/TS 60479-1, Effects of Current on Human Beings and Livestock - Part 1: General Aspects, International Electrotechnical Committee: Geneva, 2005.

[4] Friedlander, G.D., Electricity in hospitals: elimination of lethal hazards. IEEE Spectrum, 8(9), pp. 40-51, 1971. doi: http://dx.doi.org/10.1109/MSPEC.1971.5218422

[5] Amicucci, G.L., Di Lollo, L., Fiamingo, F., Mazzocchi, V., Platania, G., Ranieri, D., Razzano, R., Camin, G., Sebastiani, G. \& Gentile, P., Electrical safety during transplantation. Transplantation Proceedings, 42, pp. 2175-2180, 2010. doi: http:// dx.doi.org/10.1016/j.transproceed.2010.05.133

[6] IEC EN 60601-1, Medical Electrical Equipment - General Requirements for Basic Safety and Essential Performance, 3rd edn, International Electrotechnical Committee: Geneva, 2007.

[7] Watson, A.B., Wright, J.S. \& Loughman, J., Electrical thresholds for ventricular fibrillation in man. Medical Journal of Australia, 24(1), pp 1179-1182, 1973.

[8] Laks, M., Arzbaecher, R., Bailey, J., Berson, A., Briller, S. \& Geselowitz, D., Will relaxing safe current limits for electromedical equipment increase hazards to patients? Circulation, 89(2), pp. 909-910, 1994. doi: http://dx.doi.org/10.1161/01.CIR.89.2.909

[9] Swerdlow, C.D., Olson, W.H., O’Connor, M.E., Gallik, D.M., Malkin, R.A. \& Laks, M., Cardiovascular collapse caused by electrocardiographically silent $60-\mathrm{Hz}$ intracardiac leakage current: implications for electrical safety. Circulation, 99, pp. 2559-2564, 1999. doi: http://dx.doi.org/10.1161/01.CIR.99.19.2559

[10] Guidance for Industry and FDA Premarket and Design Control Reviewers; U.S Dept. of Health and Human Services, Food and Drug Administration, Medical Device UseSafety: Incorporating Human Factors Engineering into Risk Management, U.S Dept. of Health and Human Services, the Food and Drug Administration: USA, 2000.

[11] Amicucci, G.L., Fiamingo, F. \& Mazzetti, C., The Hospital Electrical Power Systems: Design and Operation Guidelines (in Italian Gli impianti elettrici ospedalieri: indicazioni costruttive e di utilizzo), "Gestire la sicurezza di impianti e tecnologie biomediche, Proposte per l'innovazione tecnologica in ambito sanitario", Monografico ISPESL, Supplemento a Prevenzione Oggi n. 1, INAIL: Rome, 2008, ISBN 9788889415444. 
[12] Amicucci, G.L., Fiamingo, F., Zennaro, E. \& Poggi, L., Risk management for the protection and safety of electrical systems involving healthcare buildings (in Italian Gestione del rischio per la protezione e la sicurezza dei sistemi elettrici a servizio delle strutture ospedaliere), VGR 2012, Valutazione e Gestione del Rischio negli Insediamenti Civili ed Industriali, Pisa, October 3-5, 2012.

[13] IEC 60364-7-710, Electrical Installations of Buildings: Part 7-710, Requirements for Special Installations or Locations: Medical Locations, International Electrotechnical Committee: Geneva, 2002.

[14] EN ISO 14971, Medical Devices - Application of Risk Management to Medical Devices, 2nd edn., 2012.

[15] Spalding, L., Carpes, W.Jr. \& Batistela, N., A method to detect the microshock risk during a surgical procedure. IEEE Transactions on Instrumentation and Measurement, 58(7), pp. 2335-2342, 2009. doi: http://dx.doi.org/10.1109/TIM.2009.2013925

[16] IEC EN 62353, Medical Electrical Equipment - Recurrent Test and Test After Repair of Medical Electrical Equipment, International Electrotechnical Committee: Geneva 2008.

[17] Joint Committee for Guides in Metrology, Evaluation of measurement data - supplement 1 to the "guide to the expression of uncertainty in measurement" - propagation of distributions using a Monte Carlo method, Technical report, Joint Committee for Guides in Metrology, 2008. 